\title{
Transfers in the Group of Multiplicative Units of the Classical Cohomology Ring and Stiefel-Whitney Classes
}

By

\author{
Andrzej KozLowsKI*
}

\begin{abstract}
It is proved that $G(X)$-the group of multiplicative units of the classical cohomology ring $\prod_{i \geqq 0} H^{\imath}(X ; Z / 2)$ of a $C W$-complex $X$ admits a transfer map $N_{\pi}^{w}: G(X) \rightarrow G(Y)$ defined for finite coverings $\pi: X \rightarrow Y$, such that total Stiefel-Whitney class $w: K O() \rightarrow G()$ is a transfer commuting natural transformation. It is also shown that $N_{\pi}^{w}$ possesses all the properties of transfers in generalized cohomology theories and for double coverings can be expressed in terms of the Evens transfer ("Evens norm").
\end{abstract}

\section{§ 0. Introduction}

The principal purpose of this paper is to answer a question I raised in [9]. Namely, consider the total Stiefel-Whitney class as a homomorphism

$$
w: \widetilde{K O}(X) \longrightarrow G(X ; Z / 2)
$$

where $G(X ; Z / 2)$ denotes the group of multiplicative units of the classical cohomology ring

$$
\mathscr{H}(X)=\prod_{i=0}^{\infty} H^{\imath}(X ; Z / 2)
$$

of a $C W$-complex $X$. In [9] it was shown that the functor $G(X ; Z / 2)$ possesses a "transfer homomorphism" defined for double coverings $X \rightarrow Y$ such that the diagram

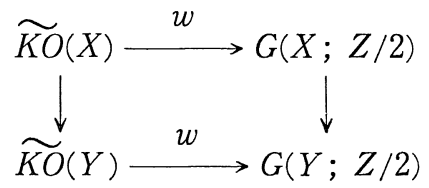

where the vertical arrows denote transfers, is commutative. (Actually in [9] this was proved only for the augmented Stiefel-Whitney class

Communicated by N. Shimada, May 26, 1988.

* Department of Mathematics, Wayne State University, Detroit, Michigan 48202, U.S.A. 


$$
\hat{w}: K O(X) \longrightarrow \hat{G}(X)
$$

where $\hat{G}(X ; Z / 2)=H^{0}(X ; Z) \times G(X ; Z / 2)$ and $\hat{w}=(\operatorname{rank}, w)$, but as will be shown later, these statements are equivalent). In cases like this we shall say that the total Stiefel-Whitney class commutes with the transfer, and sometimes, that the transfer commutes with the total Stiefel-Whitney class. In [9] I asked if the definition of the above transfer can be extended to arbitrary finite coverings, so that it possess all the "usual properties". It is in fact easy to see that the answer is yes, the transfer is in fact in a certain sense induced by the one in KO-theory. Unfortunately the way the transfer is defined does not appear to throw much light on the question of whether it is the transfer in some generalized cohomology theory $G^{*}(X ; Z / 2)$ for which $G^{0}(X ; Z / 2)=G(X ; Z / 2)$.

The definition of the transfer and verification of its properties occupies the first three sections of the paper. The last section is devoted to pointing out various relationships between this work (and some of my past work) and the papers of Fulton \& Macpherson [6] and B. Kahn [7].

This paper was written while the author was visiting the Tokyo Institute of Technology. I would like to thank the members of the Department of Mathematics, and especially professors Y. Sasao, T. Fukuda and K. Yamaguchi for helping to make my stay both enjoyable and productive.

\section{$\S 1$. The Main Theorem}

Our main result is the following theorem.

Theorem 1.1. For each finite covering $\pi: X \rightarrow Y$ there is a transfer map $N_{\pi}^{w}: G(X ; Z / 2) \rightarrow G(Y ; Z / 2)$ such that the diagram

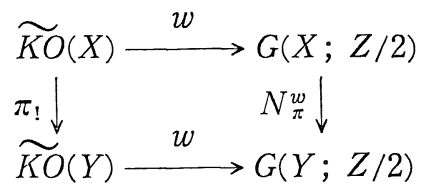

is commutative (where $\pi_{!}$denotes the transfer in reduced $K$-theory). This transfer is uniquely characterized by the following properties:

1. If

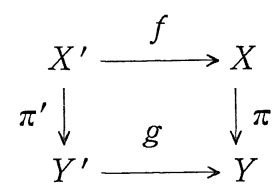

is a homotopy fibre square with $f$ and $g$ finite coverings then

$$
N_{\pi}^{w} \circ f^{*}=g^{*} \circ N_{\pi}^{w} .
$$


2. If $\pi=1: X \rightarrow X$ then $N_{\pi}^{w}=1: G(X ; Z / 2) \rightarrow G(X ; Z / 2)$.

3. If $\underset{r}{\rightarrow \rightarrow} \stackrel{p}{\rightarrow} Z$ is a composite covering then $N_{p \circ r}^{w}=N_{p}^{w} \circ N_{r}^{w}$.

4. If $\pi: X \rightarrow Y$ is a disjoint union of coverings $X=X_{1} \cup X_{2}$ and if $x \in$ $G(X ; Z / 2)$ then

$$
N_{\pi}^{w}(x)=N_{\pi_{1}}^{w}\left(i_{1}^{*} x\right) \cup N_{\pi_{2}}^{w}\left(i_{2}^{*} x\right)
$$

where $i_{k}: X_{k} \rightarrow X$ is the inclusion and $\pi_{k}=\left.\pi\right|_{X_{k}}$ the restriction for $k=1,2$.

5. Let $\pi: X \rightarrow Y$ be a double covering. Then

$$
N_{\pi}^{w}\left(\sum_{i=0}^{\infty} x_{i}\right)=N_{\pi}\left(\sum_{i=0}^{\infty} x_{i}\right)+\sum_{i=0}^{\infty} N_{\pi}\left(x_{i}\right)\left((1+t)^{-i}+1\right)
$$

where $N_{\pi}$ denotes the Evens transfer (or rather its analogue for cohomology with $Z / 2$ coefficients, see [3], [6], [7], [11], [16],), and $t=w_{1}\left(\pi_{!}\left(1_{X}\right)\right)$.

Properties 1-4 imply that $N_{\pi}^{w}$ is a homomorphism (consider the covering $X \cup X \rightarrow X \rightarrow Y$ ). One can also deduce a "double coset" formula in the manner of [16] p. 130.

It is clear that properties $1-5$ of $N_{\pi}^{w}$ characterize it uniquely because of the following well known result, which is a special case of Proposition 12.1 of [6].

Proposition 2.1. Let $\pi: X \rightarrow Y$ be a covering with $Y$ connected. Then there is a connected covering $Y^{\prime} \rightarrow Y$ of odd degree such that the induced covering $\underset{Y}{X} Y^{\prime} \rightarrow Y^{\prime}$ is a disjoint union of coverings $X_{\alpha} \rightarrow Y^{\prime}$, each of which is either an isomorphism or factors into a composite of double coverings.

\section{$\S 2$. Construction of $N_{\pi}^{w}$}

It is well known that $H^{*}(B O ; Z / 2)=Z / 2\left[w_{1}, w_{2}, \cdots\right]$, where $B O$ is the classifying space for stable orthogonal real vector bundles and the $w_{\imath}$ 's are the universal Stiefel-Whitney classes. $B O$ is of course an $H$-space with respect to the Whitney sum

$$
\oplus: B O \times B O \longrightarrow B O
$$

and, moreover, an infinite loop space. This implies that there is an $E_{\infty}$-operad $\{L(n)\}_{n \geqq 0}$ which acts on $B O$ via a family of Dyer-Lashof maps

$$
D_{B O}^{n}: L(n) \underset{\Sigma_{n}}{\times} B O^{n} \longrightarrow B O
$$

(e. g. [12]). Since each $L(n)$ is a free contractible $\Sigma_{n}$-space (where $\Sigma_{n}$ denotes the symmetric group on $n$ symbols), we shall often use the notation $E \Sigma_{n}$ in place of $L(n)$. We shall also assume from now until further notice that all cohomology groups have coefficients in $Z / 2$ and write $H^{n}(X), G(X)$, in place of $H^{n}(X ; Z / 2), G(X ; Z / 2)$, etc. We shall also assume that all $C W$-complexes are connected, although this assumption is not essential as will become clear later. 
It is clear that there is an isomorphism of sets

$$
G(X) \cong \mathscr{H} \circ \mathrm{m}\left(H^{*}(B O), H^{*}(X)\right)
$$

where $\left.\mathcal{H} \circ \mathrm{m}^{*}(B O), H^{*}(X)\right)$ denotes the set of degree preserving ring homomorphisms, given by

$$
1+x_{1}+x_{2}+\cdots \leftrightarrow f
$$

where $f\left(w_{\imath}\right)=x_{\imath}$. We next make the right hand side of $(A)$ into an abelian group by defining the sum of two ring homomorphisms $f, g: H^{*}(B O) \rightarrow H^{*}(X)$ to be the composite homomorphism

$$
\begin{aligned}
H^{*}(B O) \stackrel{\oplus}{\longrightarrow} H^{*}(B O \times B O) & \cong H^{*}(B O) \otimes H^{*}(B O) \stackrel{f \otimes g}{\longrightarrow} H^{*}(X) \otimes H^{*}(X) \\
& \cong H^{*}(X \times X) \stackrel{\Delta^{*}}{\longrightarrow} H^{*}(X)
\end{aligned}
$$

where $\Delta: X \rightarrow X \times X$ is the diagonal inclusion. With this abelian group structure on the right (A) becomes an isomorphism of abelian groups. The homomorphism

$$
\begin{gathered}
\widetilde{K O}(X)=[X, B O] \longrightarrow \mathscr{H} \circ\left(H^{*}(B O), H^{*}(X)\right) \cong G(X) \\
f \longrightarrow f^{*} \longrightarrow 1+f^{*} w_{1}+f^{*} w_{2}+\cdots
\end{gathered}
$$

is, of course, the total Stiefel-Whitney class. (These simple observations, which nevertheless play a key role in what follows, I learned from [14]. I would like to thank Kenshi Ishiguro for bringing this paper to my attention).

Let now $\phi: H^{*}(X) \rightarrow H^{*}(Y)$ be a ring homomorphism (where $X$ and $Y$ are locally finite $C W$-complexes). Let $S$ be a subgroup of the symmetric group $\Sigma_{n}$. Then $\phi$ induces a homomorphism of rings $T_{S} \phi: H^{*}\left(E S \underset{S}{\times} X^{n}\right) \rightarrow H^{*}\left(E S \underset{S}{\times} Y^{n}\right)$. Moreover, if $\phi$ is induced by a map of spaces $f: Y \rightarrow X$, i. e. $\phi=f^{*}$, then $T_{S} f^{*}$ is induced by the map of spaces $1 \times f^{n}: E S \underset{S}{\times} X^{n} \rightarrow E S \underset{S}{\times Y^{n}}$. The correspondence $\phi \rightarrow T_{S} \phi$ is natural with respect to homomorphisms $S^{\prime} \rightarrow S^{\prime \prime}$ of subgroups of $\Sigma_{n}$. All of this follows from the work of Nakaoka ([13] Theorem 3.3) as generalized to spaces in [7]. Namely, there is an isomorphism of rings

$$
H^{*}\left(E S \underset{S}{\times} X^{n}\right) \cong H^{*}\left(S ; H^{*}(X)^{\otimes n}\right)
$$

which is natural with respect to maps of spaces and homomorphisms of groups. From this the above assertions immediately follow, but as it will be later convenient to have an explicit construction of the map

$$
T_{S}: \mathscr{H}_{o m}\left(H^{*}(X), H^{*}(Y)\right) \longrightarrow \mathscr{H} \circ{ }^{*}\left(H^{*}\left(E S \underset{\boldsymbol{S}}{\times} X^{n}\right), H^{*}\left(E S \underset{\boldsymbol{S}}{\times} Y^{n}\right)\right)
$$

by means of cochains, so we briefly describe it below. Let $\phi$ be as above. Then $\phi$ can be represented by a map of cochain complexes $\Phi: C^{*}(X) \rightarrow C^{*}(Y)$. Since the cochain complexes have coefficients in $Z / 2$ any two such maps inducing the same homomorphism on $H^{*}$ are cochain homotopic. 
Since $C^{*}\left(E S \underset{S}{\times} X^{n}\right) \cong \operatorname{Hom}_{S}\left(C_{*}(E S), C^{*}(X)^{\otimes n}\right)$, the cochain map $\Phi$ induces a cochain map

$$
\operatorname{Hom}_{S}\left(1 ; \Phi^{\otimes n}\right): \operatorname{Hom}_{S}\left(C_{*}(E S), C^{*}(X)^{\otimes n}\right) \longrightarrow \operatorname{Hom}_{S}\left(C_{*}(E S), C^{*}(Y)^{\otimes n}\right) .
$$

By a lemma of Steenrod (Lemma 3.2 in [13]), the homomorphism $H^{*}\left(\operatorname{ES} \underset{S}{\times} X^{n}\right)$ $\rightarrow H^{*}\left(E S \underset{S}{\times} Y^{n}\right)$ induced by $\operatorname{Hom}_{S}\left(1 ; \Phi^{\otimes n}\right)$ depends only on $\phi$ and thus defines $T_{S} \phi$.

When $S=\Sigma_{n}$ we shall write simply $T \phi$ for $T_{S} \phi$. We can now define the transfer $N_{\pi}^{w}$ for all finite coverings $\pi: X \rightarrow Y$ as follows. Let $x \in G(X ; Z / 2)$ be represented by a ring homomorphism $f: H^{*}(B O) \rightarrow H^{*}(X)$. Then $N_{\pi}^{w}(x)$ is represented by the ring homomorphism

$$
H^{*}(B O) \stackrel{\left(D_{B O}^{n}\right)^{*}}{\longrightarrow} H^{*}\left(E \Sigma_{n} \underset{\Sigma_{n}}{\times} B O^{n}\right) \stackrel{T f}{\longrightarrow} H^{*}\left(E \Sigma_{n} \underset{\Sigma_{n}}{\times} X^{n}\right) \stackrel{t^{*}}{\longrightarrow} H^{*}(Y)
$$

where $t: Y \rightarrow E \Sigma_{n} \underset{\Sigma_{n}}{\times} X^{n}$ is a pre-transfer map which is defined as follows. Let $\tilde{X}^{n}$ denote the subspace of $X^{n}$ given by $\tilde{X}^{n}=\left\{\left(x_{1}, \cdots, x_{n}\right) ; x_{i} \neq x_{j}\right.$ if $i \neq j$ and $\pi\left(x_{i}\right)=\pi\left(x_{j}\right)$ for all $\left.1 \leqq i, j \leqq n\right\}$. The symmetric group $\Sigma_{n}$ acts freely on $\tilde{X}^{n}$; hence we have a principal $\Sigma_{n}$ bundle $\tilde{X}^{n} \rightarrow \tilde{X}^{n} / \Sigma_{n} \approx Y$. Choose a "classifying map" of principal $\Sigma_{n}$ bundles

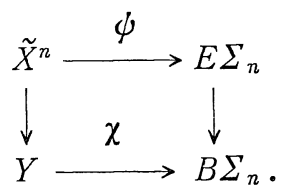

As a pre-transfer we can now take the map $t(y)=\left[\psi\left(x_{1}, \cdots, x_{n}\right),\left(x_{1}, \cdots, x_{n}\right)\right]$, where the $x_{i}$ are the $n$ points in $\pi^{-1}(y)$. It is easy to see that the homotopy classs of this map is independent of the choices made. (More details about pretransfers can be found in [1]).

\section{$\S 3$. Proof of the Main Theorem}

We will only sketch the proofs of properties 1-4 of the transfer $N_{\pi}^{w}$ since they are straight forward, and give a more detailed proof of property 5 .

1. Let

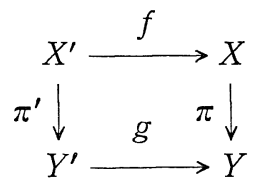

be a homotopy fibre square with $\pi: X \rightarrow Y$ and $\pi^{\prime}: X^{\prime} \rightarrow Y^{\prime}$ finite coverings. We wish to prove that

$$
N_{\pi}^{w}\left(f^{*} u\right)=g^{*}\left(N_{\pi}^{w}(u)\right)
$$


where $u \in G(X)$. Represent $u$ by a ring homomorphism $u: H^{*}(B O) \rightarrow H^{*}(X)$ 。 Then $f^{*} u$ is represented by the homomorphism

$$
f^{*} u: H^{*}(B O) \stackrel{u}{\longrightarrow} H^{*}(X) \stackrel{f^{*}}{\longrightarrow} H^{*}\left(X^{\prime}\right) .
$$

$N_{\pi}^{w}\left(f^{*} u\right)$ is represented by

$$
H^{*}(B O) \stackrel{\left(D_{B O}^{n}\right)^{*}}{\longrightarrow} H^{*}\left(E \Sigma_{n} \underset{\Sigma_{n}}{\times} B O^{n}\right) \stackrel{T\left(f^{*} u\right)}{\longrightarrow} H^{*}\left(E \Sigma_{n} \underset{\Sigma_{n}}{\times} X^{n}\right) \stackrel{t_{\pi^{\prime}}^{*}}{\longrightarrow} H^{*}\left(Y^{\prime}\right) .
$$

On the other hand $g^{*} N_{\pi}^{w}(u)$ is represented by

$$
\begin{aligned}
H^{*}(B O) & \stackrel{\left(D_{B O}^{n}\right)^{*}}{\longrightarrow} H^{*}\left(E \Sigma_{n} \underset{\Sigma_{n}}{\times} B O^{n}\right) \stackrel{T u}{\longrightarrow} H^{*}\left(E \Sigma_{n} \underset{\Sigma_{n}}{\times} X^{n}\right) \\
& \stackrel{t_{\pi}^{*}}{\longrightarrow} H^{*}(Y) \stackrel{g^{*}}{\longrightarrow} H^{*}\left(Y^{\prime}\right) .
\end{aligned}
$$

Since $T\left(f^{*} u\right)=T f^{*} \circ T u$ it is only necessary to verify that the diagram

$$
\begin{gathered}
H^{*}\left(E \sum_{n} \underset{\Sigma_{n}}{\times} X^{n}\right) \stackrel{T f^{*}}{\longrightarrow} H^{*}\left(E \sum_{n} \underset{\Sigma_{n}}{\times} X^{\prime n}\right) \\
t_{\pi}^{*}{ }_{\downarrow}^{*} g^{*}(Y) \stackrel{g_{\pi^{\prime}}^{*} \downarrow}{\longrightarrow} H^{*}\left(Y^{\prime}\right)
\end{gathered}
$$

is commutative. Since $T f^{*}=\left(\left(1 \times f^{n}\right)^{*}\right.$ this follows from the homotopy commutativity of the diagram

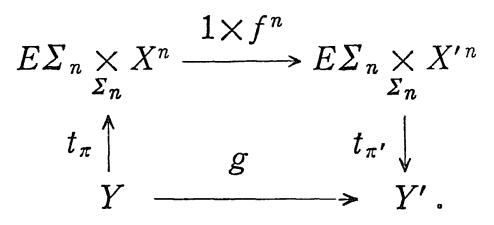

2. Obvious.

3. Let $X \stackrel{r}{\rightarrow} Y \stackrel{p}{\rightarrow} Z$ be a composite covering and let $x \in G(X)$. We want to show that

$$
N_{p \circ r}^{w}(x)=N_{p}^{w}\left(N_{r}^{w}(x)\right) .
$$

The left hand side is represented by

$$
\begin{gathered}
H^{*}(B O) \stackrel{\left(D_{B O}^{n m}\right)^{*}}{\longrightarrow} H^{*}\left(E \Sigma_{n m} \underset{\Sigma_{n m}}{\times} B O^{n m}\right) \stackrel{T_{m n} x}{\longrightarrow} \\
H^{*}\left(E \Sigma_{n m} \underset{\Sigma_{n m}}{\times} X^{n m}\right) \stackrel{t_{p \circ r}}{\longrightarrow} H^{*}(Z)
\end{gathered}
$$

where $m$ is the degree of $r$ and $n$ the degree of $-p$. The right hand side is represented by 


$$
\begin{aligned}
& H^{*}(B O) \stackrel{\left(D_{B O}^{m}\right)^{*}}{\longrightarrow} H^{*}\left(E \Sigma_{m} \underset{\Sigma_{m}}{\times} B O^{m}\right) \stackrel{T_{m}\left(D_{B O}^{n}\right)^{*}}{\longrightarrow} H^{*}\left(E \Sigma_{m} \underset{\Sigma_{m}}{\times}\left(E \Sigma_{n} \underset{\Sigma_{n}}{\times} B O^{n}\right)^{m}\right) \\
& \stackrel{T_{m} T_{n} x}{\longrightarrow} H^{*}\left(E \Sigma_{m} \underset{\Sigma_{m}}{\times}\left(E \Sigma_{n} \underset{\Sigma_{n}}{\times} X^{n}\right)^{m}\right) \stackrel{T_{m} t_{r}^{*}}{\longrightarrow} H^{*}\left(E \Sigma_{m} \underset{\Sigma_{m}}{\times} Y^{m}\right) \stackrel{t_{p}^{*}}{\longrightarrow} H^{*}(Z) .
\end{aligned}
$$

To show that the left hand side = right hand side we only need to check that the following diagrams commute

$$
\begin{aligned}
& H^{*}\left(E \Sigma_{m} \underset{\Sigma_{m}}{\times} B O^{m}\right) \stackrel{T_{m}\left(D_{B O}^{n}\right)^{*}}{\longrightarrow} H^{*}\left(E \Sigma_{m} \underset{\Sigma_{m}}{\times}\left(E \Sigma_{n} \underset{\Sigma_{n}}{\times} B O^{n}\right)^{m}\right) \\
& \left(D_{B O}^{m}\right) * \uparrow \quad\left(D_{B O}^{n m}\right)^{*} \quad\left(\nabla_{m, n}^{B O}\right) * \uparrow \\
& H^{*}(B O) \longrightarrow H^{*}\left(E \Sigma_{n m} \underset{\Sigma_{n m}}{\times} B O^{n m}\right) \\
& H^{*}\left(E \Sigma_{m} \underset{\Sigma_{m}}{\times}\left(E \Sigma_{n} \underset{\Sigma_{n}}{\times} B O^{n}\right)^{m}\right) \stackrel{T_{m} T_{n} x}{\longrightarrow} H^{*}\left(E \Sigma_{m} \underset{\Sigma_{m}}{\times}\left(E \Sigma_{n} \underset{\Sigma_{n}}{\times} X^{n}\right)^{m}\right) \\
& \begin{array}{c}
\left(\nabla_{m, n}^{B O}\right)^{*} \downarrow \\
H^{*}\left(E \Sigma_{n m} \underset{\Sigma_{n m}}{\times} B O^{n m}\right) \stackrel{T_{m n} x}{\longrightarrow} H^{*}\left(E \Sigma_{n m} \underset{\Sigma_{n m}}{\times} X^{n m}\right)
\end{array} \\
& H^{*}\left(E \Sigma_{m} \underset{\Sigma_{m}}{\times}\left(E \Sigma_{n} \underset{\Sigma_{n}}{\times} X^{n}\right)^{m}\right) \stackrel{T_{m} t_{r}^{*}}{\longrightarrow} H^{*}\left(E \Sigma_{m} \underset{\Sigma_{m}}{\times} Y^{m}\right)
\end{aligned}
$$

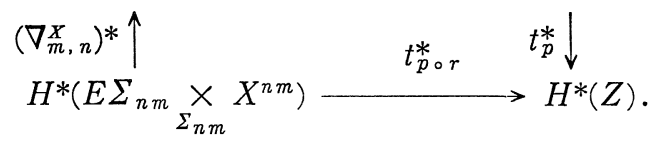

(3)

In the above diagrams the maps

$$
\left(\nabla_{m, n}^{X}\right): E \Sigma_{m} \underset{\Sigma_{m}}{\times}\left(E \Sigma_{n} \underset{\Sigma_{n}}{\times} X^{n}\right)^{m} \longrightarrow E \Sigma_{n m} \underset{\Sigma_{n m}}{\times} X^{n m}
$$

are defined using the "structure maps"

$$
\mu_{m, n_{1}, \cdots, n_{m}}: E \Sigma_{m} \times E \Sigma_{n_{1}} \times \cdots \times E \Sigma_{n_{m}} \longrightarrow E \Sigma_{n_{1}+\cdots+n_{m}}
$$

(in our case $n_{1}=n_{2}=\cdots=n_{m}=n$ ) of the operad $\left\{E \Sigma_{n}\right\}_{n \geqq 0}$. Diagram (1) commutes because the diagram

$$
\begin{gathered}
E \Sigma_{m} \underset{\Sigma_{m}}{\times}\left(E \Sigma_{n} \underset{\Sigma_{n}}{\times} B O^{n}\right)^{m} \stackrel{1 \times\left(D_{B O}^{n}\right)^{m}}{\longrightarrow} E \Sigma_{m} \underset{\Sigma_{m}}{\times} B O^{m} \\
\quad D_{m, n}^{B O} \downarrow \\
E \Sigma_{n m} \underset{\Sigma_{n m}}{\times} B O^{n m} \stackrel{D_{B O}^{n m}}{\longrightarrow} B O
\end{gathered}
$$

commutes (by definition of an operad). To see that diagram (2) commutes we consider it at the level of cochains. Ii is then necessary to show that the diagram 


$$
\begin{aligned}
& \operatorname{Hom}_{\Sigma_{m}}\left(C_{*}\left(E \Sigma_{m}\right), \operatorname{Afom}_{\Sigma_{n}}\left(C_{*}\left(E \Sigma_{n}\right), C^{*}(B O)^{(n)}\right)^{(m)}\right) \longrightarrow \\
& \operatorname{Hom}_{m}\left(C_{*}\left(E \Sigma_{m}\right), \mathcal{H}_{o m \Sigma_{n}}\left(C_{*}\left(E \Sigma_{n}\right), C^{*}(X)^{(n)}\right)^{(m)}\right) \\
& \mathscr{H}_{o m \Sigma_{m n}}\left(C_{*}\left(E \Sigma_{m n}\right), C^{*}(B O)^{(n m)}\right) \longrightarrow \mathcal{H}_{o m \Sigma_{m n}}\left(C_{*}\left(E \Sigma_{m n}\right), C^{*}(X)^{(n m)}\right)
\end{aligned}
$$

commutes (where the maps denoted by the arrows are the obvious ones). It is easy to see that this diagram can be identified with the commutative diagram

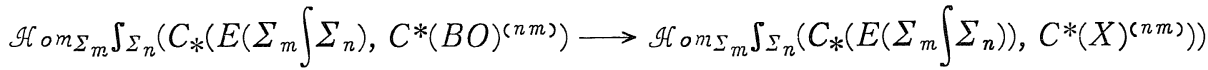

$$
\begin{aligned}
& \mathscr{H}_{\circ n \Sigma_{m n}}\left(C_{*}\left(E \Sigma_{m n}\right), C^{*}(B O)^{(n m)}\right) \longrightarrow \overbrace{\mathcal{H}^{(n m} \Sigma_{m n}}\left(C_{*}\left(E \Sigma_{m n}\right), C^{*}(X)^{(n m)}\right)
\end{aligned}
$$

in which the vertical maps are induced by the inclusions $\Sigma_{m} \int \Sigma_{n} \rightarrow \Sigma_{m n}\left(\Sigma_{m} \int \Sigma_{n}\right.$ denotes the wreath product of symmetric groups). Finally, diagram (3) commutes because the diagram

$$
\begin{aligned}
& E \Sigma_{m} \underset{\Sigma_{m}}{\times}\left(E \Sigma_{n} \underset{\Sigma_{n}}{\times} X^{n}\right)^{m} \stackrel{1 \times t_{r}}{\longleftarrow} E \Sigma_{m} \underset{\Sigma_{m}}{\times} Y^{m} \\
& E \Sigma_{n m} \underset{\Sigma_{n m}}{\times} X^{n m} \leftarrow t_{p \circ r} \quad t_{p} \downarrow
\end{aligned}
$$

is obviously homotopy commutative.

4. The proof is similar to that of 3 , and will be omitted.

5. Observe first that both sides of the identity define transfers (for double coverings) which commute with the total Stiefel-Whitney class. Indeed, for the left hand side this is what we have just proved. For the right hand side it can be proved in exactly the same way as was done in [9] in the augmented (non-reduced) case. In [9] an augmented transfer

$$
\hat{N}_{\pi}^{w}: \hat{G}(X) \longrightarrow \hat{G}(Y) \quad\left(\hat{G}(X)=H^{0}(X ; Z) \times G(X)\right)
$$

was defined for double coverings $\pi: X \rightarrow Y$ by the formula

$$
\hat{N}_{\pi}^{w}\left(m, \sum_{i=0}^{\infty} x_{i}\right)=\left(2 m, N_{\pi}\left(\sum_{i=0}^{\infty} x_{i}\right)+\sum_{i=0}^{\infty} N_{\pi}\left(x_{i}\right)\left((1+t)^{m-i}+1\right)\right)
$$

(where by convention $x_{0}=1$ ). It was also shown that the augmented transfer has the right properties and that it commutes with the augmented total StiefelWhitney class

$$
\hat{w}(\xi)=(\operatorname{rank}(\xi), w(\xi)): \hat{G}(X) \longrightarrow K O(X) 。
$$

In fact this entire paper could have been written for the non-reduced case. First, for a locally finite (not necessarily connected) $C W$-complex $X$ we can show that $\hat{G}(X)=H^{0}(X ; Z) \times G(X)=\mathscr{A}_{\text {om }}$ coalgebra $\left(H_{*}(Z \times B O), H_{*}(X)\right)$. The natural map $K O(X)=[X, Z \times B O] \rightarrow \mathcal{H}_{o m_{\text {coalgebra }}}\left(H_{*}(Z \times B O), H_{*}(X)\right)$ turns out to be the augmented total Stiefel-Whitney class $\widehat{w}(\xi)=(\operatorname{rank}(\xi), w(\xi))$ 。 Next we can now 
define the augmented transfer $\hat{N}_{\pi}^{w}$ analogously to the way $N_{\pi}^{w}$ was denined. The statement of Theorem 1.1 remains valid with $N_{\pi}^{w}$ replaced by $\hat{N}_{\pi}^{w}$, except that the formula in 5 has to be replaced by the one given in [9]. The expression in 5 in the reduced case can be deduced from the non-reduced one by making use of a curious formula discovered by B. Kahn [7]. Let us consider this in detail.

Restricting $\hat{N}_{\pi}^{w}$ to the "zero component" $G(X)=0 \times G(X) \subset \hat{G}(X)$ we get the "transfer" for double coverings given by the right hand side of the formula in 5. Let us show that the total (non-augmented) Stiefel-Whitney class commutes with this transfer. Since the augmented total Stiefel-Whitney class commutes with the augmented transfer we have:

$$
\begin{aligned}
(0, w & \left.\left(\pi_{!}\left(\xi-1^{k}\right)\right)\right)=\left(0, w(\pi !(\xi)) / w\left(\pi_{!}\left(1^{k}\right)\right)\right)=\left(2 k, w\left(\pi_{!}(\xi)\right) /\left(2 k, w\left(\pi_{!}\left(1^{k}\right)\right)\right)\right. \\
= & \left(2 k, N_{-}\left(\sum_{i=0}^{k} w_{\imath}(\xi)\right)+\sum_{i=0}^{k} N_{\pi}\left(w_{\imath}(\xi)\right)\left((1+t)^{k-i}+1\right)\right) /\left(2 k,(1+t)^{k}\right) \\
= & \left(0, N_{\pi}\left(\sum_{i=0}^{k} w_{\imath}(\xi)\right)(1+t)^{-k}+\sum_{i=0}^{k} N_{\pi}\left(w_{\imath}(\xi)\right)\left((1+t)^{k-i}+1\right)(1+t)^{-k}\right) \\
= & \left(0,\left\{N_{-}\left(\sum_{i=0}^{k} w_{\imath}(\xi)\right)(1+t)^{-k}+\sum_{i=0}^{k} N_{\pi}\left(w_{\imath}(\xi)\right)(1+t)^{-k}\right\}\right. \\
& \left.+\sum_{i=0}^{k} N_{\pi}\left(w_{\imath}(\xi)\right)\left((1+t)^{-i}\right)\right) .
\end{aligned}
$$

By Proposition 1.2.5. b of [7] we have $t N_{\pi}(x+y)=t N_{\pi}(x)+t N_{\pi}(y)$ and hence

$$
\begin{aligned}
& N_{-}\left(\sum_{i=0}^{k} w_{\imath}(\xi)\right)(1+t)^{-k}+\sum_{i=0}^{k} N_{\pi}\left(w_{\imath}(\xi)\right)(1+t)^{-k}=N_{\pi}\left(\sum_{i=0}^{k} w_{\imath}(\xi)\right) \\
& +\sum_{i=0}^{k} N_{\pi}\left(w_{\imath}(\xi)\right) .
\end{aligned}
$$

Substituting we finally obtain

$$
\left(0, w\left(\pi_{!}\left(\xi-1^{k}\right)\right)\right)=\left(0, N_{\pi}\left(\sum_{i=0}^{k} w_{\imath}(\xi)\right)+\sum_{i=0}^{k} N_{\pi}\left(w_{\imath}(\xi)\right)\left((1+t)^{-i}+1\right)\right)
$$

as required.

We have shown that both sides of the formula in property 5 define transfers for double coverings which commute with the total Stiefel-Whitney class. Let us denote them by $N_{\pi}^{w}$ (as before) and $\tilde{N}_{\pi}^{w}$. We will show that they coincide. First observe that both transfers are defined as composites of maps

with the pre-transfer

$$
D_{X}^{w}, \tilde{D}_{X}^{w}: G(X) \longrightarrow G\left(E \sum_{2} \underset{\Sigma_{2}}{\times} X^{2}\right)
$$

$$
t_{\pi}^{*}: G\left(E \Sigma_{2} \underset{\Sigma_{2}}{\times} X^{2}\right) \longrightarrow G(Y) .
$$

Thus it is enough to show that $D_{X}^{w}=\tilde{D}_{X}^{w}$. In fact it suffices to show this for 
$X=B O$. Indeed, the map $D_{X}^{w}$ can be defined in terms of $D_{B O}^{w}$ as follows. Let $x \in G(X)$ be represented by a ring homomorphism $f: H^{*}(B O) \rightarrow H^{*}(X)$. Let $w \in$ $G(B O)$ be the universal total Stiefel-Whitney class $1+w_{1}+w_{2}+\cdots$ (represented by the homomorphism 1: $\left.H^{*}(B O) \rightarrow H^{*}(B O)\right)$. We have $D_{B O}^{w}(w) \in G\left(E \Sigma_{2}{ }_{\Sigma_{2}} B O^{2}\right)=$ $\mathscr{H} o m\left(H^{*}(B O), \quad H^{*}\left(E \Sigma_{2} \underset{\Sigma_{2}}{\times} B O^{2}\right)\right)_{0} \quad$ Composing with $T f: H^{*}\left(E \Sigma_{2} \underset{\Sigma_{2}}{\times} B O^{2}\right) \rightarrow$ $H^{*}\left(E \Sigma_{2} \underset{\Sigma_{2}}{\times} X^{2}\right)$ we obtain the element $D_{X}^{w}(x) \in G\left(E \Sigma_{n} \underset{\Sigma_{2}}{\times} X^{2}\right)=\mathscr{H} \circ{ }^{*}\left(H^{*}(B O)\right.$, $\left.H^{*}\left(E \Sigma_{2} \underset{\Sigma_{2}}{\times} X^{2}\right)\right)$. Similarly we can show that $\tilde{D}_{X}^{w}(x)=T f \circ \widetilde{D}_{B O}^{w}(w)$. Indeed, we have

$$
\widetilde{D}_{B O}^{w}\left(\sum_{i=0}^{\infty} w_{i}\right)=D_{B O}\left(\sum_{i=0}^{\infty} w_{i}\right)+\sum_{i=0}^{\infty} D_{B O}\left(w_{\imath}\right)\left((1+t)^{-i}+1\right) .
$$

Applying $T f$ and using the cochain construction of $D_{X}$ (see [7] where it is denoted by $P_{2}$ ) we easily check that

$$
T f \circ \tilde{D}_{B O}^{w}\left(\sum_{i=0}^{\infty} w_{i}\right)=D_{X}\left(\sum_{i=0}^{\infty} x_{i}\right)+\sum_{i=0}^{\infty} D_{X}\left(x_{i}\right)\left((1+t)^{-i}+1\right)=\tilde{D}_{X}^{w}(x)
$$

as required. Thus it remains to show that $D_{B O}^{w}=\tilde{D}_{B O}^{w}$. Consider the covering $X \approx E \Sigma_{2} \times X \stackrel{\pi_{X}}{\rightarrow} B \Sigma_{2} \times X$. As a pre-transfer map for this covering we can take "the diagonal" $\Delta: X \rightarrow E \Sigma_{2} \underset{\Sigma_{0}}{\times} X^{2}$ (e.g. see [11]). Since is well known that the map

$$
G\left(E \Sigma_{n}{\underset{\Sigma}{n}}_{n} X^{n}\right) \stackrel{\left(i^{*}, \Delta^{*}\right)}{\longrightarrow} G\left(X^{2}\right) \oplus G\left(B \Sigma_{2} \times X\right)
$$

is injective (where $i: X^{2} \rightarrow E \Sigma_{2} \underset{\Sigma_{2}}{\times} X^{2}$ is the inclusion of a fibre for the bundle $\left.E \Sigma_{2} \underset{\Sigma_{2}}{\times} X^{2} \rightarrow B \Sigma_{2}\right)$ it suffices to show that

1. $i^{*}\left(D_{B O}^{w}\right)(w)=i^{*}\left(\tilde{D}_{B O}^{w}\right)(w)$

and

2. $\Delta^{*}\left(D_{B O}^{w}\right)(w)=\Delta^{*}\left(\widetilde{D}_{B O}^{w}\right)(w)$.

It is easy to verify that 1 holds. To prove that 2 is also true we observe that $\Delta^{*}\left(D_{B O}^{w}\right)(w)=N_{\pi_{B O}}^{w}(w), \Delta^{*}\left(\tilde{D}_{B O}^{w}\right)(w)=\tilde{N}_{\pi_{B O}}^{w}(w)$. Since $N_{\pi}^{w}$ and $\tilde{N}_{\pi}^{w}$ commute with the total Stiefel-Whitney class we have $N_{\pi_{B O}}^{w}(w(\xi))=\tilde{N}_{\pi_{B O}}^{w}(w(\xi))$ for any vector bundle $\xi$ on $B O$. Hence we must have $N_{\pi_{B O}}^{w}(w)=\tilde{N}_{\pi_{B O}}^{w}(w)$ which completes our proof.

\section{§4. Some Remarks on the Work of Fulton-MacPherson [6] and B. Kahn [7]}

The questions considered in this paper originated with the problem proposed by Atiyah in [2], of finding a purely algebraic definition of the Chern classes 
of a complex and Stiefel-Whitney classes of a real representation of a finite group. Atiyah pointed out that for this purpose it is sufficient to obtain a "purely algebraic" formula for the total Chern or Stiefel-Whitney class of a representation of a finite group induced from a representation of a subgroup in terms of Chern or Stiefel-Whitney classes of the given representation (of the subgroup). Such a formula for the Chern class was first found by Evens [4] in the case of a normal subgroup of prime index and one dimensional representation (classical results in representation theory imply that this is sufficient for the purpose of an "algebraic definition" asked for by Atiyah) and extended by Evens and D. Kahn to representations of arbitrary degree. An simple proof of an analogous formula for the total Stiefel-Whitney class of the direct image (transfer) of a real vector bundle for a double covering map was given independently by the present author in [9] and by B. Kahn in [7] (the argument in [7] is credited to J. Lannes). Finally Fulton and MacPherson [6] obtained a general formula (for arbitrary coverings), which we will describe below. (It may be worth pointing out that the definition of the transfer $N_{\pi}^{w}$ gives the simplest solution to Atiyah's original problem in the case of the Stiefel-Whitney class, since the definition can easily be given a "purely algebraic" form). My interest in these questions, unlike that of the above mentioned authors, derives primarily from infinite loop space theory. From this point of view we think of transfers which satisfy properties 1-4 of Theorem 1.1 as arising from "transfer spaces" which represent these functors, i. e. spaces admiting a compatible family of Dyer-Lashof maps. It is well known that infinite loop spaces are transfer spaces and infinite loop maps between infinite loop spaces commute with the transfers. The converses to these assertions are of course not true (e.g. see [8] and [10]) but hold in many naturally arising situations in topology (e.g. see [1]). This is the main justification for the question originally raised in [9]. Perhaps some light on this question may be thrown by comparing our main theorem with the main result of [6]. I will restate this result in a form which appears to be most suggestive in this context. In order to see this more clearly let us first consider another result of [6].

Let $\pi: X \rightarrow Y$ be a finite covering of degree $n$. Let $\lambda$ denote a monotone non-increasing sequence of non-negative integers $\lambda=\left(\lambda_{1}, \lambda_{2}, \cdots, \lambda_{n}\right), \lambda_{1} \geqq \lambda_{2} \geqq \cdots \geqq$ $\lambda_{n} \geqq 0$. To each such sequence $\lambda$ and an integer $a \in \lambda$ we associate auxiliary coverings $f_{\lambda}: X_{\lambda} \rightarrow Y$ and $\rho_{\lambda, a}: X_{\lambda, a} \rightarrow X$ with a homotopy fibre square of coverings

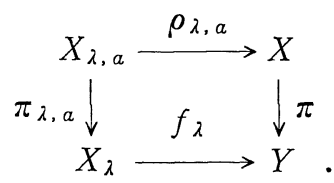

Fulton and MacPherson prove the following formula for the Evens transfer of 
a sum.

Proposition 4.1. Let $x_{\imath} \in H^{i}(X)$ for $i=1,2, \cdots$ and let $\pi: X \rightarrow Y$ be as above. Then

$$
N_{\pi}\left(\sum_{i=0} x_{\imath}\right)=\sum_{\lambda}\left(f_{\lambda}\right)_{!}\left(\prod_{a \in \lambda} N_{\pi_{\lambda}, a}\left(\rho_{\lambda, a}^{*} x_{a}\right)\right)
$$

where $\lambda$ runs over all sequences as above with $\lambda_{\imath} \geqq 1$ (The notation is as in [6] except that we denote the classical transfer with the subscript! rather than*).

In [6] the above "addition formula" is proved in a general geometric context. We remark that in the topological case an easy homotopy theoretic proof can be given, but we shall not bother to do so here.

Now we shall state the main result of [6] in a form which, in our opinion, suggests a possible inriguing relationship with the above formula. Namely, for $x \in H^{d}(X)$ define the "normalized" Evens transfer $\tilde{N}_{\pi}(x)=1 / w\left(\pi_{!}\left(1^{d}\right)\right) N_{\pi}(x)$. In words this means that the "normalized" transfer of a homogeneous element of degree $d$ is the Evens transfer of the element divided by the total StiefelWhitney class of the transfer of the trivial $d$-dimensional bundle. Of course the "normalized" transfer of a homogeneous element is not longer homogeneous. Now we define the "normalized" transfer of a sum by the formula in Proposition 4.1 with $\tilde{N}_{\pi}$ in place of $N_{\pi}$, i. e.

$$
\tilde{N}_{\pi}\left(\sum_{i=0} x_{\imath}\right)=\sum_{\lambda}\left(f_{\lambda}\right)_{1}\left(\prod_{a \in \lambda} \tilde{N}_{\pi_{\lambda}, a}\left(\rho_{\lambda, a}^{*} x_{a}\right)\right) .
$$

The main result of ([6], Theorem 5.1) can now be easily shown to be equivalent to

Theorem 4.2. Let $\pi: X \rightarrow Y$ be a finite covering. Then the "transfer" $\tilde{N}_{\pi}$ : $G(X) \rightarrow G(Y)$ commutes with the (reduced) total Stiefel-Whitney class.

It seems virtually certain that the above "transfer" is the same as the one defined in our main theorem (Theorem 1.1) i.e.

$$
\tilde{N}_{\pi}=N_{\pi}^{w}
$$

but this appears to be difficult to prove. Of course it is sufficient to show that the "transfer" defined by the Fulton-MacPherson formula possesses properties 1-5 in Theorem 1.1. In fact this is easy to show that it possesses properties $1,2,4,5$ (see [6] pp. 54-58, 90) but a direct proof of property 3 appears to be extremely complicated. It is, however, interesting to note that, at least in the topological case, one can obtain several of the consequences of the main formula of [6] by using only Theorem 1.1 and well known facts from homotopy theory and infinite loop space theory. The following results are proved in [6] as orollaries of the main formula. 
Proposition 4.3. (Corollaries 5.2 and 5.3 of [6]). Let $\pi: X \rightarrow Y$ be as above and let $E$ be a real vector bundle over $X$ of rank $e$. Then we have the following formulas:

(a) $w_{1}\left(\pi_{!} E\right)=\pi_{!}\left(w_{1}(E)\right)+e w_{1}\left(\pi_{!}\left(1_{X}\right)\right)$

(b) $w_{2}\left(\pi_{!} E\right)=\pi_{!}\left(w_{2}(E)\right)+\pi_{!}^{(2)}\left(w_{1}(E)\right)+e w_{1}\left(\pi_{!}\left(1_{X}\right)\right) \pi_{!}\left(w_{1}(E)\right)+w_{2}\left(\pi_{!}\left(1_{\dot{X}}^{e}\right)\right)$

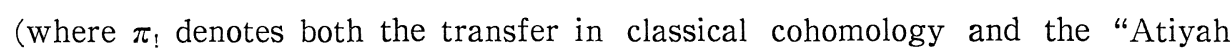
transfer" for vector bundles, while $\pi_{!}^{(2)}$ denotes the "second order" Evens transfer, see [3], [6], [7]).

Proof. First observe that we can write (a) in the form

$$
w_{1}\left(\pi_{!}\left(E-1_{X}^{\oplus e}\right)\right)=\pi_{!}\left(w_{1}\left(E-1_{X}^{\oplus e}\right)\right)
$$

which is the same as saying that the first Stiefel-Whitney class

$$
w_{1}: \widetilde{K O}(X) \longrightarrow H^{1}(X ; Z / 2)
$$

commutes with the transfer. But it is well known that the map

$$
w_{1}: B O \longrightarrow K(Z / 2 ; 1)
$$

is in fact an infinite loop map and thus commutes with the transfer. To prove (b) (and in fact simultaneously re-prove (a)) we generalize the above argument. Consider the natural homomorphism (truncated total Stiefel-Whitney class)

$$
w_{/ 3}=\left(w_{1}, w_{2}\right): \widetilde{K O}(X) \longrightarrow G_{/ 3}(X)=H^{1}(X ; Z / 2) \times H^{2}(X ; Z / 2) .
$$

Here the right hand side is an abelian group with the group operation given by $\left(x_{1}, x_{2}\right)+\left(y_{1}, y_{2}\right)=\left(x_{1}+y_{1}, x_{2}+y_{2}+x_{1} y_{1}\right)$. In fact this group is the zero-th term of a connective cohomology theory, by [15]. Indeed, in [15] it is shown that for any (anti-)commutative graded ring $A=\left\{A_{i}\right\}_{i \geqq 0}$ the $H$-space $\Pi_{\imath \geqq 1} K\left(A_{i}, i\right)$ representing the group of multiplicative units of the ring $\Pi_{i \geqq 0} H^{i}\left(X, A_{i}\right)$ is an infinite loop space. If we take $A=Z / 2[x]$-the graded ring of polynomials in one indeterminate $x$ of degree 1 , we obtain the infinite loop space structure on the representing space of the functor $G(X)$ whose corresponding transfer is the Evens transfer. If instead we take a truncated polynomial ring $A=Z / 2[x] / x^{n}$ we obtain an infinite loop space structure on the finite product of EilenbergMacLane spaces $\prod_{i=0}^{n-1} K(Z / 2 ; i)$ and in particular taking $n=3$ we obtain an infinite loop space structure on the product $K(Z / 2 ; 1) \times K(Z / 2 ; 1)$ mentioned above. The transfer corresponding to this cohomology theory is easy to describe. Let $\pi: X \rightarrow Y$ be a finite covering and let $1+x_{1}+x_{2}$ be an element of $G_{13}(X)$ (we now think of $G_{/ 3}(X)$ as the group of multiplicative units in the ring $H^{0}(X ; Z / 2)$ $\left.\times H^{1}(X ; Z / 2) \times H^{2}(X ; Z / 2)\right)$. The transfer $N_{\pi}^{\prime 3}$ in $G_{13}(X)$ is just the transfer $N_{\pi}$ in $G(X)$ truncated in degrees $\geqq 3$, thus 
(c) $N_{\pi}^{\prime 3}\left(1+x_{1}+x_{2}\right)=1+\pi:\left(x_{1}\right)+\pi_{!}\left(x_{2}\right)+\pi_{!}^{(2)}\left(x_{1}\right)$

(this follows from well known properties of the Evens transfer, and can be easily proved directly from the definition). Now, it is easy to show that formulas (a) and (b) are together equivalent to the assertion that $w_{/ 3}$ commutes with the transfer. Indeed, suppose that

$$
w_{/ 3}\left(\pi_{!}\left(E-1_{X}^{e}\right)\right)=N_{\pi}^{\prime 3}\left(w_{/ 3}\left(E-1_{X}^{e}\right)\right)
$$

then

hence

$$
w_{/ 3}\left(\pi_{!} E-\pi_{!} 1_{X}^{e}\right)=N_{\pi}^{\prime 3}\left(1+w_{1}(E)+w_{2}(E)\right)
$$

$$
\left(1+w_{1}\left(\pi_{!} E\right)+w_{2}(\pi: E)\right) /\left(1+w_{1}\left(\pi: 1_{X}^{e}\right)+w_{2}\left(\pi_{!} 1_{X}^{e}\right)\right)=N_{\pi}^{\prime 3}\left(1+w_{1}(E)+w_{2}(E)\right) .
$$

Cross-multiplying and substituting the expression (c) into the right hand side, we obtain formulas (a) and (b).

One can easily show that $w_{/ 3}$ commutes with the transfer by making use of formulas 4, 5 in Theorem 1.1 and Proposition 2.1, from which we see that $N_{\pi}=N_{\pi}^{w}$ modulo terms of degree $\geqq 3$. (I proved the corresponding result for the Chern class in this way some time ago, see the remark on p. 430-431) in [10]). However, at least in the case of the total Stiefel-Whitney class, a much stronger statement is true: $w_{/ 3}$ is an infinite loop map. A proof of this fact will appear elsewhere. Note however, that this statement is not true for any $w_{/ n}$ with $n>3$, indeed, such $w_{/ n}$ do not commute with the transfer.

As another example let us prove, by a similar method, another of the corollaries of the main formula of [6].

Proposition 4.4. (Corollary 5.7 of [6]) Suppose $k>0$ and $w_{2} E=0$ for $0<i$ $<k$. Then

$$
w_{k}\left(\pi_{!} E\right)=\pi_{!} w_{k}(E)+w_{k}\left(\pi_{!} 1_{X}^{e}\right) .
$$

Proof. Re-write the above equality in the form

$$
w_{k}\left(\pi_{!}\left(E-1_{X}^{e}\right)\right)=\pi_{!} w_{k}\left(E-1_{X}^{e}\right) .
$$

To prove this, consider the map $f: X \rightarrow B O$ which classifies the virtual bundle $E-1_{X}^{e}$. The assumption implies that $f$ lifts to a map to $(k-1)$-connected $K O$ theory

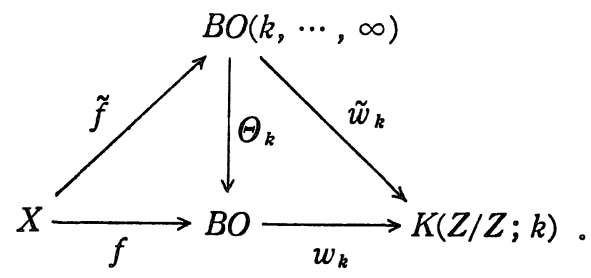


Now, it is well known that $\tilde{w}_{k}$, the lifting of $w_{k}$ to the $(k-1)$-connected cover of $B O$ is an infinite loop map, hence it commutes with the transfer, i.e.

$$
\pi_{!} \tilde{w}_{k}(\tilde{f})=\tilde{w}_{k}\left(\pi_{!} \tilde{f}\right) .
$$

(Here we are using the symbol $f$ to denote both the map and the corresponding element of $[X, B O(k, \cdots, \infty)]$. The transfer on the R.H.S. is, of course, the one corresponding to $(k-1)$-connected $K O$-theory).

Since $\Theta_{k} \tilde{f}=f$ and $\Theta_{k}$ commutes with the transfer (being an infinite loop map) we have

$$
\left.w_{k}\left(\pi_{!} f\right)=w_{k}\left(\pi_{!} \Theta_{k} \tilde{f}\right)=w_{k}\left(\Theta_{k}\left(\pi_{!} \tilde{f}\right)\right)=\tilde{w}_{k}\left(\pi_{!} \tilde{f}\right)\right)=\pi_{!} \tilde{w}_{k}(\tilde{f})=\pi_{!} w_{k}(f) .
$$

This completes the proof. Of course exactly the same argument can be used to prove the analogous result for Chern classes.

Finally, we observe that many of the results of [6] and [7] about StiefelWhitney classes of "direct image bundles" can be "generalized" as statements of properties of the transfer $N_{\pi}^{w}$, without reference to vector bundles. For example the following

Proposition 4.5. (Corollary 5.5 of [7]). Let $E$ be a real vector bundle of rank e. Let $\pi: X \rightarrow Y$ be a covering of degree $n$. Then

$$
w_{n e}(E)=N_{\pi}\left(w_{e}(E)\right)
$$

can be deduced from

Proposition 4.6. Let $\left(e, 1+x_{1}+\cdots+x_{e}\right) \in \hat{G}(X)$. Let $\hat{N}_{\pi}^{w}\left(e, 1+x_{1}+\cdots+x_{e}\right)$ $=\left(e n, P\left(e, x_{1}, \cdots, x_{e}\right)\right)$. Then the term of degree en in $P\left(e, x_{1}, \cdots, x_{e}\right)$ is $N_{\pi}\left(x_{e}\right)$.

Proof. It is well known and easy to show that the term of degree ne in $N_{\pi}\left(1+x_{1}+\cdots+x_{e}\right)$ is $N_{\pi}\left(x_{e}\right)$. Thus for a double covering $(n=2)$ the result follows from the formula

$$
\hat{N}_{\pi}^{w}\left(e, \sum_{i=0}^{e} x_{i}\right)=\left(2 e, N_{\pi}\left(\sum_{i=0}^{e} x_{i}\right)+\sum_{i=0}^{e} N_{\pi}\left(x_{i}\right)\left((1+t)^{e-i}+1\right)\right) .
$$

It now follows at once from Theorem 1.1 and well known properties of the Evens transfer $N_{\pi}([3],[6],[7],[16])$ that it is true for a composite covering and a disjoint union. The general case now follows from Proposition 2.1.

As another example, the same method proves the following formula:

Proposition 4.7. Let $d \equiv 0 \bmod 2^{k+1}$. Then $\hat{N}_{\pi}^{w}\left(d, 1+x_{2^{k}}+x_{2^{k}+1}+\cdots\right)=$ (nd, $1+\pi_{!}\left(x_{2^{k}}\right)+\cdots+\pi_{!}\left(x_{2^{k+1}-1}\right)+\left\{\right.$ terms of degree $\left.\left.\geqq 2^{k+1}\right\}\right)$.

From this Proposition 1.3.4 of [7] follows immediately. 


\section{References}

[1] J.F. Adams, Infinite loop spaces, Annals of Mathematics Studies, no. 90 (Princeton University Press, 1978).

[2] M.F. Atiyah, Characters and cohomology of finite groups, Publ. Math. I.H.E.S No. 9 (1961), 23-64.

[3] L. Evens, A generalization of the transfer map in the cohomology of groups, Trans. A. M.S. 108 (1963), 54-65.

[4] - On the Chern classes of representations of finite groups, Trans, A.M.S. 115 (1965), 180-193.

[5] L. Evens \& D. Kahn, Chern classes of certain representations of symmetric groups, Trans. A. M.S. 245 (1978), 309-330.

[6] W. Fulton \& R. MacPherson, Characteristic classes of direct image bundles for covering maps, Annals of Mathematics, 125 (1987), 1-92.

[7] B. Kahn, Classes de Stiefel-Whitney de formes quadratiques et de representations galoisiennes réelles, Invent. Math. 78 (1984), 223-256.

[8] D. Kraines \& T. Lada, A counter example to the transfer conjecture, Algebraic Topology, Waterloo, 1978, Lecture Notes in Mathematics, No. 741, Springer, New York (1979), 588-624.

[9] A. Kozlowski, The Evens-Kahn formula for the total Stiefel-Whitney class, Proc. A. M.S. 91 (1984), 309-314.

[10] - On a theorem of F. Roush, Quart. J. Math. 35 (1984), 429-438.

[11] — The transfer in Segal's cohomology, Illinois Journal of Math. 27, No. 4 (1983), 614-623.

[12] J.P. May, The geometry of iterated loop spaces, Lecture Notes in Mathematics, No. 271, Springer, New York, 1972.

[13] M. Nakaoka, Homology of the infinite symmetric group, Ann. of Math. 73(2) (1961), 229-257.

[14] R. Patterson, The square-preserving algebra endomorphisms of $H^{*}\left(B O, Z_{2}\right)$, Quart。 J. Math. Oxford (2), 29 (1978), 225-240.

[15] G.B. Segal, The multiplicative group of classical cohomology, Quart. J. Math. Oxford (2) 26 (1975), 289-293.

[16] R. Steiner, Multiplicative transfers in classical cohomology Proc. Edinburgh Math Soc. 25 (1982), 113-131. 